\title{
Remission of ALK-negative primary pulmonary inflammatory myofibroblastic tumor on treatment with clarithromycin: A case report and review of the literature
}

\author{
HIDEHIRO WATANABE ${ }^{1,2}$, TOMONORI URUMA $^{1,2}$, GEN TAZAKI $^{2}$, TAKUMA TAJIRI $^{3}$, \\ RYOTA KIKUCHI $^{1}$, MASAYUKI ITOH $^{1}$, KAZUTETSU AOSHIBA ${ }^{1}$ and HIROYUKI NAKAMURA ${ }^{1}$ \\ ${ }^{1}$ Department of Respiratory Medicine and Infection Control, Ibaraki Medical Center, \\ Tokyo Medical University, Inashiki, Ibaraki 300-0395; Departments of ${ }^{2}$ Respiratory Medicine and ${ }^{3}$ Pathology, \\ Tokai University Hachioji Hospital, Tokai University School of Medicine, Hachioji, Tokyo 192-0032, Japan
}

Received January 17, 2015; Accepted December 23, 2015

DOI: $10.3892 / \mathrm{ol} .2016 .4119$

\begin{abstract}
Inflammatory myofibroblastic tumors (IMTs) belong to an intermediate group of soft-tissue tumors, they are relatively rare but exhibit a wide range of pathologies, from benign to malignant. At present, no standard treatment has been established, however, it is known to be important to determine the grade of malignancy of the tumor, prior to treatment. The present study reports a 73-year-old female patient with no clinical manifestations, who, when examined radiographically at a health check exhibited bilateral thoracic infiltrative shadows and nodular shadows by chest CT. A metastatic tumor or an organizing pneumonia were suspected. Blood examination showed no abnormal findings, and a pathological diagnosis of IMT was given from the histological findings of the tissue extracted by video-assisted thoracic surgery. Histological analysis established the lack of expression of anaplastic lymphoma kinase (ALK1) and immunoglobulin subtype G4 (IgG4). Alteration of the radiological shadows was observed over several weeks, and after concluding that chronic inflammation was worsening the patient's condition, clarithromycin was administered as a long-term macrolide therapy. The IMT decreased in size, and eight months later it had almost resolved. The patient was last reported to be maintaining a stable condition with no relapse. Some IMT cases have malignant pathology, and should be carefully followed-up. However, in the present case, where the IMT is both ALK1-negative and IgG4-negative, its biological immune responsiveness appears
\end{abstract}

Correspondence to: Professor Hidehiro Watanabe, Department of Respiratory Medicine and Infection Control, Ibaraki Medical Center, Tokyo Medical University, 3-20-1 Chuo, Ami, Inashiki, Ibaraki 300-0395, Japan

E-mail: hw-nabe4@tokyo-med.ac.jp

Key words: inflammatory myofibroblastic tumor, anaplastic lymphoma kinase, clarithromycin, macrolide, anti-inflammatory agent to differ from positive cases, and an inflammatory response was predominant. Clarithromycin, has immunomodulatory and anti-inflammatory effects and appeared to be effective in treating the IMT of the patient in the present study.

\section{Introduction}

Inflammatory myofibroblastic tumors (IMTs) belong to an intermediate group of soft-tissue tumors, and were first reported in 1990 by Pettinato et al (1). IMTs are associated with a number of non-specific symptoms, including fever, pain and weight-loss, which are common to many diseases. IMTs are therefore difficult to differentiate clinically from malignant tumors and infections. IMTs develop in the soft tissues of patients of a wide age-range, from infants to adults, and are most commonly detected in the lung, mesentery, and omentum (2). Histological findings and pathological analysis are indispensable for diagnosing IMTs, and they are characterized by proliferation of spindle-shaped cells, including fibroblasts and myofibroblasts that stain positive for alpha-smooth muscle actin ( $\alpha$-SMA), accompanied by inflammatory cell infiltration (3). Furthermore, an increased level of the pro-inflammatory cytokine interleukin (IL)-6 in peripheral blood, and an increase in nuclear factor kappa B activation in peripheral blood mononuclear cells are both commonly detected in clinical manifestations of IMTs (4). IMTs are frequently removed by surgery, and complete resection often results in a good prognosis (5).

In recent years, associations with anaplastic lymphoma kinase 1 (ALK1, commonly referred to as ALK) gene rearrangements and the presence of immunoglobulin subclass G4 (IgG4)-expressing plasma cells have been highlighted as important factors for differential diagnosis of IMTs. ALK expression has been observed in $\sim 50 \%$ of IMTs (6), and ALK-positive IMTs are reported to have high rates of rapid progression and recurrence, associated with a high grade of malignancy $(7,8)$. Associations between IgG4-positive IMTs and autoimmune diseases appear to have been suggested (9). Pathologically, infiltration by lymphocytes and plasma cells, in addition to spindle-shaped cells, is observed, and they are considered to be inflammatory pseudotumors (IPTs) (10). 
IgG4 is detected in IPTs but seldom in IMTs, whereas ALK expression has not been detected in IPTs. Thus, ALK and IgG4 expression may be regarded as important for the classification of IMTs $(11,12)$. The present study reports the first case of pulmonary IMT without ALK and IgG4 to show an improvement in the patient's condition with use of clarithromycin as a long-term macrolide therapy.

\section{Case report}

A 73-year-old female with a past medical history of hystero-oophorectomy for uterine cancer at 60 years of age, who had never smoked, was examined in our department (Respiratory Medicine and Infection Control, Tokai University Hachioji Hospital, Tokyo, Japan) on November 12, 2012 after abnormal chest shadows were observed during a health check-up with a radiographer. The patient had no fever or cough, and no particular clinical manifestations were observed. Additionally, no superficial lymph nodes were palpable, and no skin rashes were noted. Chest radiography and computed tomography (CT) revealed infiltrative shadows primarily in the left pulmonary hilum, and small nodular shadows in both the right and left lung fields (Figs. 1 and 2). The blood examination and tumor marker results were within normal limits (CEA $2.0 \mathrm{ng} / \mathrm{mL}$, CYFRA $1.1 \mathrm{ng} / \mathrm{mL}$, RProGRP $31.6 \mathrm{pg} / \mathrm{mL}$, NSE $8.7 \mathrm{ng} / \mathrm{mL}$, sIL-2R $327 \mathrm{U} / \mathrm{mL}$ and KL-6 $410 \mathrm{IU} / \mathrm{mL})$; the interferon- $\gamma$ release assay for active tuberculosis (IGRA: T-SPOT.TB) was negative, and neither sputum cultures or cytology yielded any significant findings. Two weeks following the first relevant examination (FRE), a transbronchial lung biopsy (TBLB) was performed on the patient on November 26, 2012, who was admitted to the Tokai University Hachioji Hospital overnight. Histopathological assessment of the biopsy tissue revealed nonspecific organized tissue with no evidence of malignancy. No clinical manifestations were observed during that time; however, a definitive diagnosis was deemed necessary, and after consulting with the patient and obtaining consent, video-assisted thoracic surgery (VATS) and excision of the right upper nodule was performed on December 18, 2012, three weeks after the TBLB, for which the patient was admitted until December 28, 2012. Histological evaluation of the frozen material demonstrated the presence of an organizing pneumonia with no malignancy. A detailed pathological evaluation of fixed tissue led to the preliminary diagnosis of an IgG4-related, solitary, fibrous IMT, which required further immunohistochemical investigation. The immunostaining revealed that the nodule was vimentin-positive, strongly $\alpha$-SMA-positive, CD34-negative, CD68-positive, Congo-red-negative, ALK-negative and IgG4-negative (Fig. 3). No evidence of malignancy, including evidence of cancer or spindle-cell carcinoma, was detected. The macroscopic findings showed a clearly demarcated mass. Based on the above findings, a final diagnosis of IMT was made. As there were no clinical manifestations, we consulted with the patient and decided to carefully follow her course. The chronic inflammation in the ALK-negative tissue was considered to be a chronic respiratory tract inflammation at that time. Clarithromycin was administered as a long-term macrolide antibiotic therapy, three months following the FRE, as we expected it would be effective in controlling the inflammation. The chest shadows slowly resolved on follow-up imaging examinations, performed every two months. The chest shadows had almost completely disappeared on the images obtained eight months following clarithromycin therapy (Figs. 1 and 2). Clarithromycin therapy was performed for ten months in total, and during this time there were no clinical manifestations and no impairments to the patient's everyday life. The patient has experienced no relapses, and appears to have followed a favorable course almost one year following the FRE.

\section{Discussion}

IMTs are characterized histologically by the proliferation of spindle-shaped cells (fibroblasts and myofibroblasts) and an accompanying infiltration by inflammatory cells, including lymphocytes, plasma cells, and histiocytes. The histological findings in the present case revealed relatively uniform spindle-shaped cells, and immunohistochemical staining demonstrated that the cells were vimentin-positive, strongly $\alpha$-SMA-positive, CD68-positive, CD34-negative, IgG4-negative, and both Congo-red-negative and Direct-fast-scarlet (DFS)-negative, showing the absence of amyloid (Fig. 3). Our findings were consistent with the description of earlier IMT cases by Makhlouf et al (13) and Coffin et al (6), and we therefore made a diagnosis of IMT. The behavior of IMTs range from benign to malignant, and they have been treated using a number of different methods, including surgery, anticancer drug therapy, and steroids (14-16). However, it has been reported that some cases are made worse by the use of steroids (17). To date, no standard treatment has been established. Furthermore, there are a number of reports that ALK-positive IMTs have a poor prognosis $(7,8,18)$, contrasting with relatively favorable reports for ALK-negative cases (19). However, Coffin et al (6) reported that ALK-reactivity was associated with local recurrence, but not distant metastasis (which was confined to ALK-negative lesions). This was based on the analysis of 59 cases of IMTs, of which $56 \%$ were ALK-positive. The absence of ALK expression was associated with a greater age overall, and death from disease or distant metastases. Thus, ALK reactivity may be a favorable prognostic indicator in IMT.

There have been several previous reports of successful treatment of ALK-positive IMTs with the ALK inhibitor crizotinib (20-22). Gene- and immunological-classifications according to ALK, IgG4 and other markers, as well as histological classifications, appear necessary for selecting among treatment options for IMT. Crizotinib may become the mainstay of treatment for ALK-positive cases.

The etiology of IMT implies that an inflammatory response is important to the pathology, and IMT cases have often been presented clinically as a fever of unknown origin $(23,24)$. Local proinflammatory cytokine production has been previously demonstrated to have an impact on the pathology (4). Kuppe et al (25) reported that macrophage activation syndrome was seen in IMT of the lung and thoracic spine. Accumulation and activation of macrophages by the inflammatory processes of IMT led to phagocytosis of hematopoietic stem cells in the bone marrow and caused an increase in proinflammatory cytokines, including IL-1 $\beta$, IL-6, and sIL-2R, in the blood.

In the present case, the patient's IMT was ALK-negative, and controlling the inflammatory response appeared to lead to improvement of the pathology. Chaves et al (26) investigated 
A

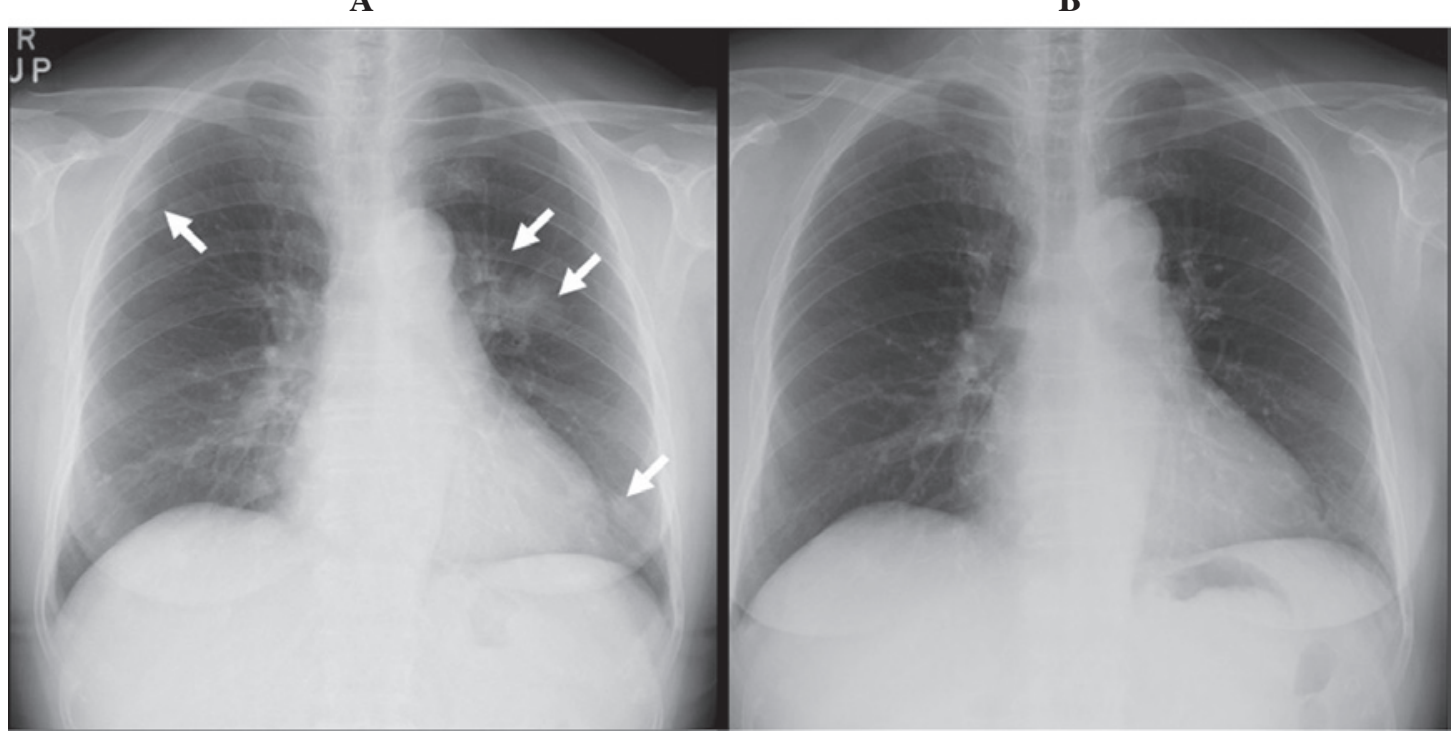

Figure 1. Chest radiographs (A) prior to and (B) following clarithromycin treatment. (A) At the time of the first relevant examination (FRE), infiltration shadows near the aorta and left lower field, and a small nodular shadow in upper right lung field were observed (arrows). (B) Nine months following initiation of clarithromycin treatment ( 1 year following FRE), the infiltrative shadows have improved and have almost disappeared.

A
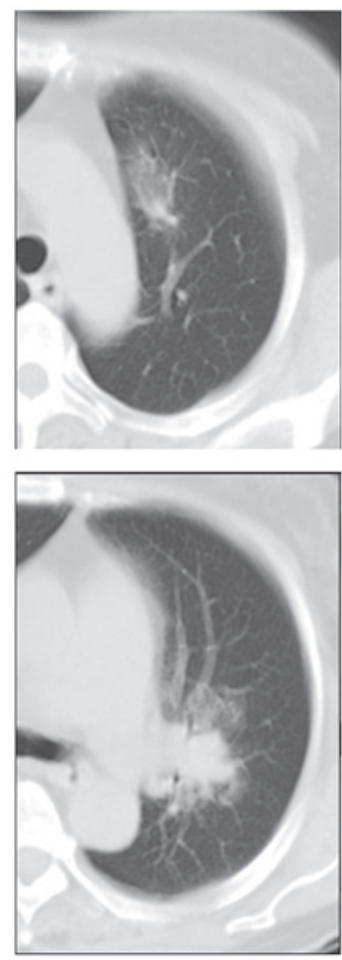

B
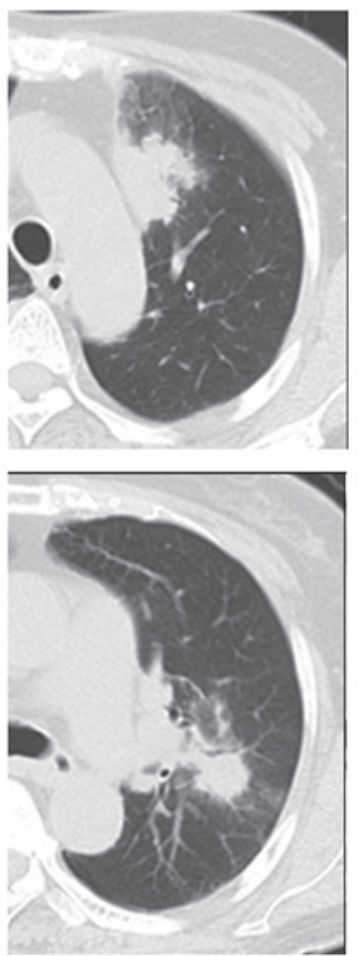

C
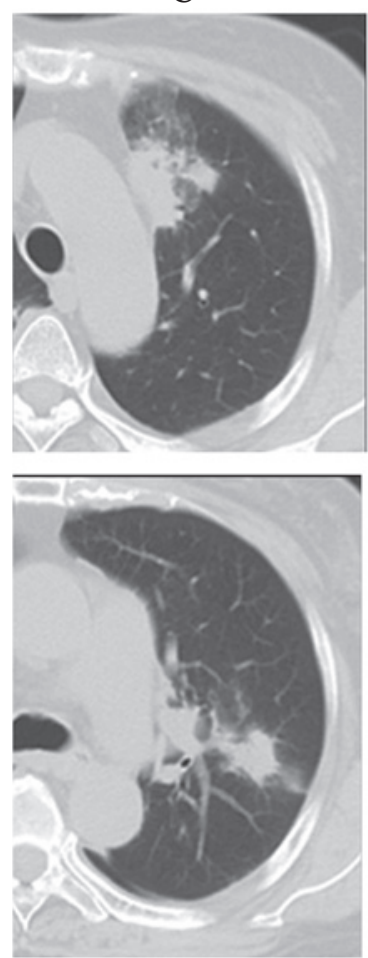

D
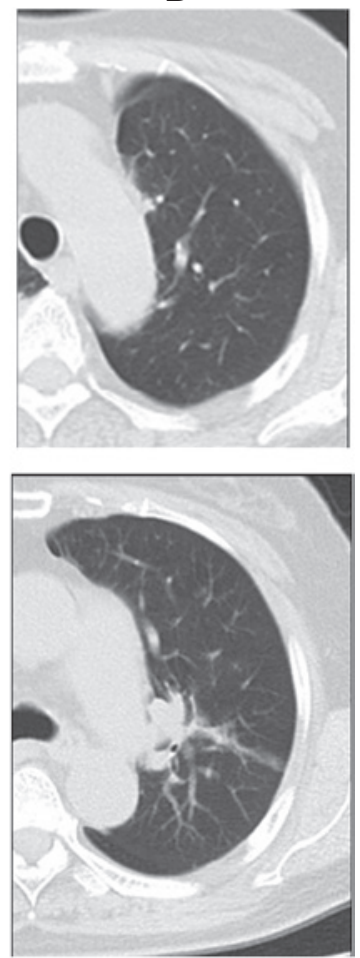

Figure 2. Chest computed tomography (CT) imaging during the clinical course. At the time of the first relevant examination (FRE), infiltrative shadows were observed near the aorta and the left-lung hilus (A, upper and lower panels). The shadows increased and a portion developed into nodules (B, upper and lower panels) two months following FRE. Five months following FRE (two months following initiation of clarithromycin treatment), the shadows disappeared gradually (C, upper and lower panels). One year following FRE (nine months following treatment), the infiltrative shadows had almost disappeared and had improved (D, upper and lower panels). Upper panels, CT images at the level of the aorta. Lower panels, CT images near the hilus of the left lung.

the anti-inflammatory action of non-steroidal anti-inflammatory drugs (NSAIDs). The authors analyzed reports of ALK-negative cases receiving NSAID therapy and reported the striking result that NSAIDs were effective in treating 10 of 11 cases of IMT.
Some macrolide antibiotics are known to possess unique anti-inflammatory activities (27). Siddiqui et al (28) reported the ability of macrolides to reduce the secretion of proinflammatory cytokines, ameliorate the infiltration of inflammatory 
A
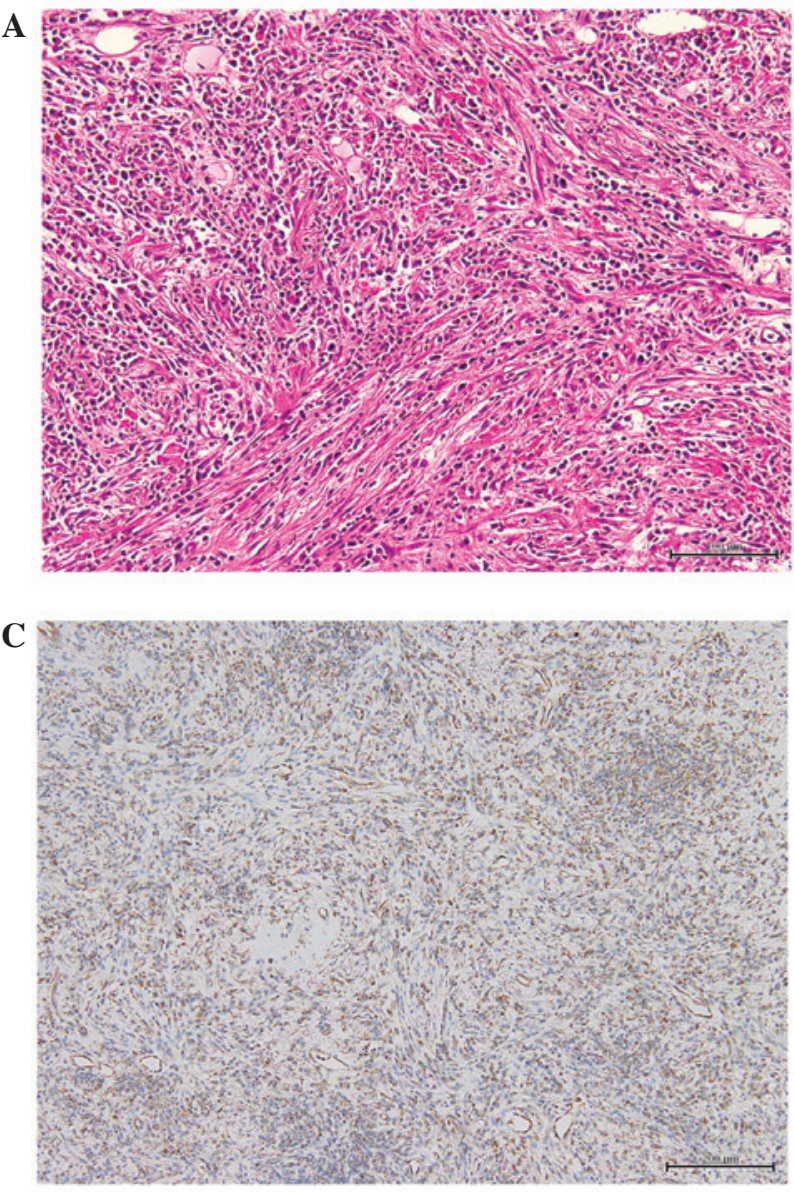

E

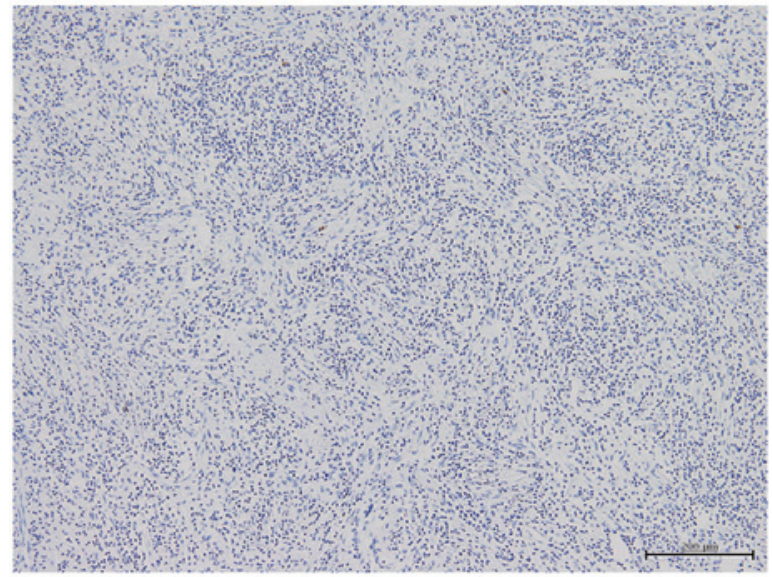

B

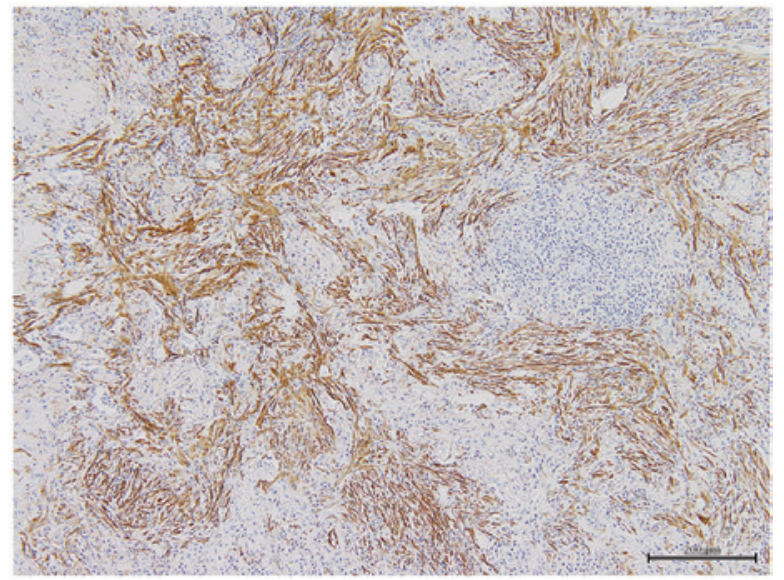

D

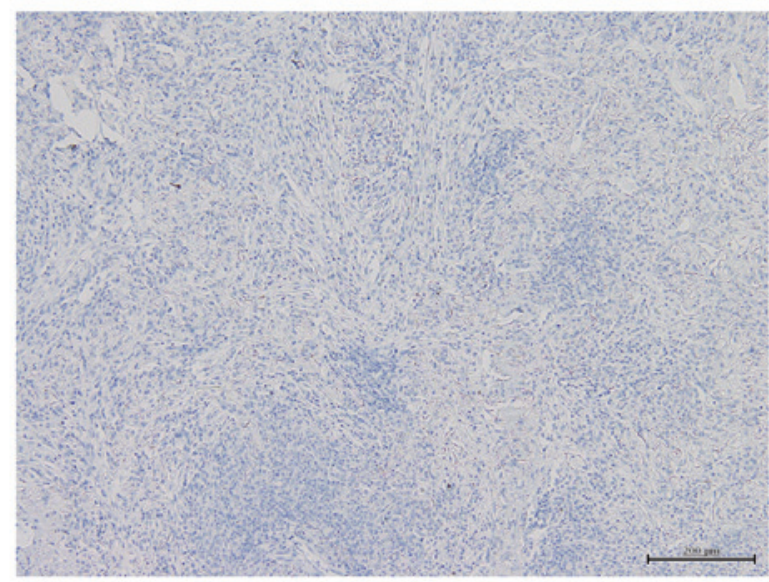

F

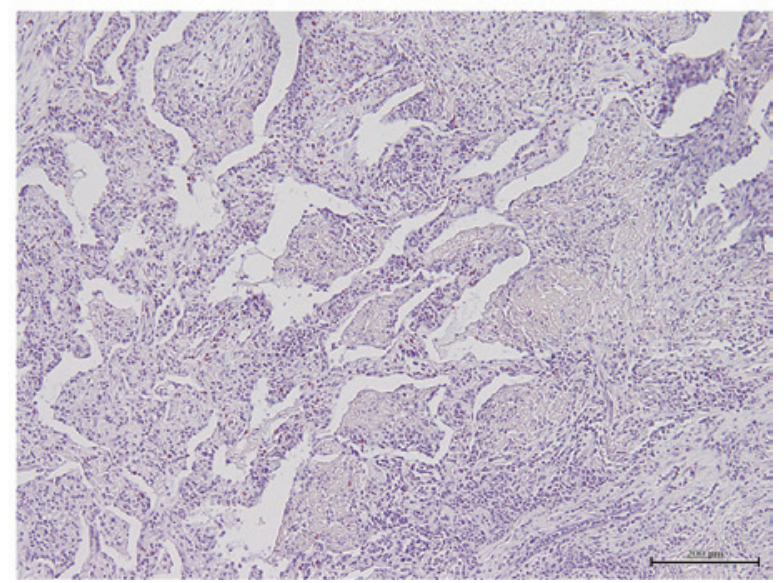

Figure 3. Micrographs of the excised inflammatory myofibroblastic tumor with histological analyses. (A) Hematoxylin and Eosin staining, representative of the center of the lesion and characterized by haphazardly-arranged spindle cells in an inflamed stroma (Scale bar, $100 \mu \mathrm{m}$ ). (B) Spindle cells with strong reactivity for alpha-smooth-muscle-actin (Scale bar, $100 \mu \mathrm{m}$ ). (C) Positivity for vimentin (Scale bar, $200 \mu \mathrm{m}$ ). (D) No staining detected for anaplastic lymphoma kinase (Scale bar, $200 \mu \mathrm{m}$ ). (E) No staining detected for IgG4 (Scale bar, $200 \mu \mathrm{m}$ ). (F) No staining detected with direct fast scarlet (Scale bar, $200 \mu \mathrm{m}$ ).

cells into the airways, and to reduce mucus secretion, enables them to improve pulmonary function and the quality of life for patients with chronic inflammatory diseases of the airways (cystic fibrosis, asthma, bronchiectasis, panbronchiolitis and cryptogenic organizing pneumonia), as well as diffuse panbronchiolitis. Furthermore, macrolide antibiotics decrease levels of IL-1 $\beta$, IL-6, IL-10, tumor necrosis factor-alpha, the chemokine ligands (CCLs) 33, 5, 20 and 22, the CXC chemokine ligands CXCL1, CXCL5, and granulocyte-colony stimulating factor in the sputum cells from patients with chronic obstructive lung disease, and have a local anti-inflammatory effect (29-31). Macrolide antibiotics have been effective in other inflammatory diseases aside from respiratory diseases, including adult Still's disease (32), rheumatoid arthritis (33), and Crohn's disease (34).

Since tissue inflammation appears to be the central pathology when an IMT is both ALK-negative and IgG4-negative, controlling the inflammation seems to be an important factor in improving the pathology. The suppression of inflammatory cell accumulation and suppression of the production of cytokines, including Il- 6 and IL-8, by macrolide antibiotics confers 
anti-inflammatory immunosuppressive effects that differ from the effects of NSAIDs, and furthermore, macrolides appear to be effective against chronic tissue inflammation.

To the best of our knowledge, there have been no previous reports of the treatment of ALK-negative, IgG4-negative IMTs with macrolide drugs, including clarithromycin, and the present case appears to be the first reported case of IMT treated with a macrolide. It may therefore be worth performing a trial of anti-inflammatory treatment of a series of ALK-negative, IgG4-negative IMTs in a future study

In conclusion, IMTs are rare disease entity, sometimes with malignant pathology, and careful attention to the diagnosis and treatment remains essential. In cases where IMTs are ALK-negative and IgG4-negative, the anti-inflammatory-immunomodulating effects of macrolide antibiotics may have a potential benefit. It is possible that ALK- and, IgG4-negative IMTs will be reclassified in the future, based on more detailed genetic analysis and immunohistochemical studies of the lesion.

\section{References}

1. Pettinato G, Manivel JC, De Rosa N and Dehner LP: Inflammatory myofibroblastic tumor (plasma cell granuloma). Clinicopathologic study of 20 cases with immunohistochemical and ultrastructural observations. Am J Clin Pathol 94: 538-546, 1990.

2. Zhao JJ, Ling JQ, Fang Y, Gao XD, Shu P, Shen KT, Qin J, Sun YH and Qin XY: Intra-abdominal inflammatory myofibroblastic tumor: Spontaneous regression World J Gastroenterol 20 13625-13631, 2014.

3. World Health Organization Classification of Tumors: Inflammatory myofibroblastic tumor. Pathology and Genetics of Tumors of Soft Tissue and Bone. IARC Press, Lyon, pp91-93, 2002.

4. Fukano R, Matsubara T, Inoue T, Gondo T, Ichiyama $\mathrm{T}$ and Furukawa S: Time lag between the increase of IL- 6 with fever and NF-kappaB activation in the peripheral blood in inflammatory myofibroblastic tumor. Cytokine 44: 293-297, 2008

5. Sakurai H, Hasegawa T, Watanabe S, Suzuki K, Asamura H and Tsuchiya R: Inflammatory myofibroblastic tumor of the lung. Eur J Cardiothorac Surg 25: 155-159, 2004.

6. Coffin CM, Hornick JL and Fletcher CD: Inflammatory myofibroblastic tumor: Comparison of clinicopathologic, histologic, and immunohistochemical features including ALK expression in atypical and aggressive cases. Am J Surg Pathol 31: 509-520, 2007.

7. Sokai A, Enaka M, Sokai R, Mori S, Mori S, Gunji M, Fujino M and Ito M: Pulmonary inflammatory myofibroblastic tumor harboring EML4-ALK fusion gene. Jpn J Clin Oncol 44: 93-96, 2014.

8. Denis DJ, Elayoubi K, Weil AG, Berthelet F and Bojanowski MW: Inflammatory myofibroblastic tumors of the central nervous system that express anaplastic lymphoma kinase have a high recurrence rate. Surg Neurol Int 4: 70, 2013.

9. Karashima T, Taniguchi Y, Shimamoto T, Nao T, Nishikawa $H$ Fukata S, Kamada M, Inoue K, Oko K, Nakajima $\mathrm{H}$, et al: IgG4-related disease of the paratestis in a patient with Wells syndrome: A case report. Diagn Pathol 9: 225, 2014.

10. Yamamoto H, Yamaguchi H, Aishima S, Oda Y, Kohashi K, Oshiro $\mathrm{Y}$ and Tsuneyoshi M: Inflammatory myofibroblastic tumor versus IgG4-related sclerosing disease and inflammatory pseudotumor: A comparative clinicopathologic study. Am J Surg Pathol 33: 1330-1340, 2009.

11. Zen Y, Kitagawa S, Minato H, Kurumaya H, Katayanagi K, Masuda S, Niwa H, Fujimura M and Nakanuma Y: IgG4-positive plasma cells in inflammatory pseudotumor (plasma cell granuloma) of the lung. Hum Pathol 36: 710-717, 2005.

12. Bhagat P, Bal A, Das A, Singh N and Singh H: Pulmonary inflammatory myofibroblastic tumor and IgG4-related inflammatory pseudotumor: A diagnostic dilemma. Virchows Arch 463: 743-747, 2013.

13. Makhlouf HR and Sobin LH: Inflammatory myofibroblastic tumors (inflammatory pseudotumors) of the gastrointestinal tract: How closely are they related to inflammatory fibroid polyps? Hum Pathol 33: 307-315, 2002.
14. Dishop MK, Warner BW, Dehner LP, Kriss VM, Greenwood MF, Geil JD and Moscow JA: Successful treatment of inflammatory myofibroblastic tumor with malignant transformation by surgical resection and chemotherapy. J Pediatr Hematol Oncol 25: $153-158,2003$.

15. Lee MH, Lee HB, Lee YC, Rhee YK, Lee EJ, Chung MJ, Jin GY, Kweon EY and Park SJ: Bilateral multiple inflammatory myofibroblastic tumors of the lung successfully treated with corticosteroids. Lung 189: 433-435, 2011.

16. Diop B, Konate I, Ka S, Sall I, Fall D, Dieng M and Wone Y: Mesenteric myofibroblastic tumor: NSAID therapy after incomplete resection. J Visc Surg 148: e311-e314, 2011.

17. Schaeffer CJ,Minai OA, Sharma N, Kanne JP and Mohammed TL: Inflammatory myofibroblastic tumor of the lung: Recurrence after steroid treatment. J Thorac Imaging 23: 191-193, 2008.

18. He CY, Dong GH and Liu HG: Recurrent laryngeal inflammatory myofibroblastic tumor with positive anaplastic lymphoma kinase mimicking recurrent respiratory papillomatosis: A case report. World J Surg Oncol 12: 54, 2014.

19. Satomi T, Watanabe M, Matsubayashi J, Nagao T and Chiba H: A successfully treated inflammatory myofibroblastic tumor of the mandible with long-term follow-up and review of the literature. Med Mol Morphol 43: 185-191, 2010.

20. Lovly CM, Gupta A, Lipson D, Otto G, Brennan T, Chung CT, Borinstein SC, Ross JS, Stephens PJ, Miller VA, et al: Inflammatory myofibroblastic tumors harbor multiple potentially actionable kinase fusions. Cancer Discov 4: 889-895, 2014.

21. Jacob SV, Reith JD, Kojima AY, Williams WD, Liu C and Vila Duckworth L: An unusual case of systemic inflammatory myofibroblastic tumor with successful treatment with ALK-Inhibitor. Case Rep Pathol 2014: 470340, 2014.

22. Tothova $Z$ and Wagner AJ: Anaplastic lymphoma kinase-directed therapy in inflammatory myofibroblastic tumors. Curr Opin Oncol 24: 409-413, 2012

23. Qiu JF, Shi YJ, Fang L, Wang HF and Zhang MC: High fever as an initial symptom of primary gastric inflammatory myofibroblastic tumor in an adult woman. Int J Clin Exp Med 7: 1468-1473, 2014

24. Zhou R, Xiang J, Chen Z, Li Z and Hong J: Fever of unknown origin as a presentation of colonic inflammatory myofibroblastic tumor in a 36-year-old female: A case report. Oncol Lett 7: 1566-1568, 2014

25. Kuppe C, Westphal S, Bücher E, Moeller MJ, Heintz B, Schneider ME and Floege J: Macrophage activation syndrome in a patient with pulmonary inflammatory myofibroblastic tumour. Allergy Asthma Clin Immunol 8: 6, 2012.

26. Chavez C and Hoffman MA: Complete remission of ALK-negative plasma cell granuloma (inflammatory myofibroblastic tumor) of the lung induced by celecoxib: A case report and review of the literature. Oncol Lett 5: 1672-1676, 2013.

27. Tamaoki J, Kadota J and Takizawa H: Clinical implications of the immunomodulatory effects of macrolides. Am J Med 117 (Suppl 9A): 5S-11S, 2004.

28. Siddiqui J: Immunomodulatory effects of macrolides: Implications for practicing clinicians. Am J Med 117 (Suppl 9A): 26S-29S, 2004

29. Giamarellos-Bourboulis EJ: Macrolides beyond the conventional antimicrobials: A class of potent immunomodulators. Int $\mathrm{J}$ Antimicrob Agents 31: 12-20, 2008.

30. Zarogoulidis P, Papanas N, Kioumis I, Chatzaki E, Maltezos $\mathrm{E}$ and Zarogoulidis K: Macrolides: From in vitro anti-inflammatory and immunomodulatory properties to clinical practice in respiratory diseases. Eur J Clin Pharmacol 68: 479-503, 2012.

31. Marjanović N, Bosnar M, Michielin F, Willé DR, Anić-Milić T, Culić O, Popović-Grle S, Bogdan M, Parnham MJ and Eraković Haber V: Macrolide antibiotics broadly and distinctively inhibit cytokine and chemokine production by COPD sputum cells in vitro. Pharmacol Res 63: 389-397, 2011.

32. Thanou-Stavraki A, Aberle T, Aksentijevich I, Bane BL and Harley JB: Clarithromycin in adult-onset still's disease: A potentially useful therapeutic. J Clin Rheumatol 17: 373-376, 2011.

33. Urasaki Y, Nori M, Iwata S, Sasaki T, Hosono O, Kawasaki H, Tanaka H, Dang NH, Ikeda E and Morimoto C: Roxithromycin specifically inhibits development of collagen induced arthritis and production of proinflammatory cytokines by human $\mathrm{T}$ cells and macrophages. J Rheumatol 32: 1765-1774, 2005.

34. Gui GP, Thomas PR, Tizard ML, Lake J, Sanderson JD and Hermon-Taylor J: Two-year-outcomes analysis of Crohn's disease treated with rifabutin and macrolide antibiotics. J Antimicrob Chemother 39: 393-400, 1997. 